\title{
Dopaminergic Genetic Variation Influences Aripiprazole Effects on Alcohol Self-Administration and the Neural Response to Alcohol Cues in a Randomized Trial
}

\author{
Joseph P Schacht ${ }^{*, 1}$, Konstantin E Voronin', Patrick K Randall' and Raymond F Anton' \\ 'Department of Psychiatry and Behavioral Sciences, Medical University of South Carolina, Charleston, SC, USA
}

\begin{abstract}
Dopamine (DA) signaling regulates many aspects of Alcohol Use Disorder (AUD). However, clinical studies of dopaminergic medications, including the DA partial agonist aripiprazole (APZ), have been inconsistent, suggesting the possibility of a pharmacogenetic interaction. This study examined whether variation in DA-related genes moderated APZ effects on reward-related AUD phenotypes. The interacting effects of APZ and a variable number tandem repeat (VNTR) polymorphism in DATI/SLC6A3 (the gene encoding the DA transporter (DAT)) were tested. In addition, interactions between APZ and a genetic composite comprising the DATI VNTR and functional polymorphisms in catechol-O-methyltransferase (COMT), DRD2, and DRD4 were evaluated. Ninety-four non-treatment-seeking individuals with AUD were genotyped for these polymorphisms, randomized to APZ (titrated to $15 \mathrm{mg}$ ) or placebo for 8 days, and underwent an fMRI alcohol cue-reactivity task (day $7 ; n=81$ ) and a bar lab paradigm (day 8). Primary outcomes were alcohol cue-elicited ventral striatal (VS) activation and the number of drinks consumed in the bar lab. DATI genotype significantly moderated medication effects, such that APZ, relative to placebo, reduced VS activation and bar-lab drinking only among carriers of the DATI 9-repeat allele, previously associated with lower DAT expression and greater reward-related brain activation. The genetic composite further moderated medication effects, such that APZ reduced the primary outcomes more among individuals who carried a larger number of DATI, COMT, DRD2, and DRD4 alleles associated with higher DA tone. Taken together, these data suggest that APZ may be a promising AUD treatment for individuals with a genetic predisposition to higher synaptic DA tone.

Neuropsychopharmacology (2018) 43, 1247-1256; doi: I0. I038/npp.20 17.298; published online 24 January 2018
\end{abstract}

\section{INTRODUCTION}

Dopamine (DA) signaling regulates many aspects of Alcohol Use Disorder (AUD). Alcohol cues and intravenous alcohol self-administration both increase DA release in the human ventral striatum (VS) (Boileau et al, 2003; Oberlin et al, 2013). Individuals with AUD, relative to controls, display enhanced alcohol-induced (Yoder et al, 2016) but blunted amphetamine-induced VS DA release (Martinez et al, 2005) and, unlike controls, demonstrate no association between striatal DA release and prefrontal glucose metabolism (Volkow et al, 2007), suggesting impaired cortical modulation of VS DA signaling. To remediate this impairment, several dopaminergic medications have been explored as AUD treatments, including the atypical antipsychotic aripiprazole (APZ) (Vergne and Anton, 2010), a highaffinity $\mathrm{D}_{2}, \mathrm{D}_{3}$, and $5-\mathrm{HT}_{1 \mathrm{~A}}$ partial agonist that also acts as an antagonist at $5-\mathrm{HT}_{2 \mathrm{~A}}, 5-\mathrm{HT}_{2 \mathrm{~B}}, 5-\mathrm{HT}_{2 \mathrm{C}}$, and $5-\mathrm{HT}_{7}$ receptors (Shapiro et al, 2003; Yokoi et al, 2002). Despite

\footnotetext{
*Correspondence: Dr JP Schacht, Department of Psychiatry and Behavioral Sciences, Medical University of South Carolina, 67 President Street, MSC 86I, Charleston, SC 29425, USA, Tel: + I 843792 7497, Fax: + I 843792 7353, E-mail: schacht@musc.edu

Received 9 August 2017; revised 27 November 2017; accepted 2 December 2017; accepted article preview online 6 December 2017
}

this relatively broad pharmacological profile, APZ is of particular interest for AUD, because its $\mathrm{D}_{2}$ partial agonist property may stabilize dysregulated DA neurotransmission by increasing striatal DA synthesis among individuals with low basal synthesis capacity and decreasing it among individuals with high basal capacity (Ito et al, 2012).

APZ has been reported to reduce alcohol-induced euphoria (Kranzler et al, 2008), drinking in the natural environment and a bar-lab setting (Voronin et al, 2008), and alcohol cue-elicited VS activation (Myrick et al, 2010). In a large multisite AUD clinical trial, APZ did not significantly change the primary drinking outcome (percent days abstinent), but did significantly improve other outcomes, including drinks per drinking day and an alcohol consumption biomarker (Anton et al, 2008). As in other studies, these latter effects were highly variable, suggesting that betweensubjects differences in an unknown factor might moderate APZ effects.

Given APZ's effects on DA transmission, one such between-subjects difference might be DA-related genetic variation. The DA transporter (DAT) is the primary mechanism for striatal DA clearance. A $40 \mathrm{bp}$ variable number tandem repeat (VNTR) polymorphism (rs28363170) in the 3'-untranslated region of the DAT gene (DAT1/ SLC6A3), whose most common allelic variants are 9 and 10 
repeats, may affect DAT function. The 9-repeat (9R) allele, relative to the 10 -repeat (10R) allele, has been associated with reduced DAT expression (Fuke et al, 2001) and, among AUD individuals, lower striatal DAT availability (Heinz et al, 2000), potentially leading to relatively increased extrasynaptic DA tone. Consistent with these findings, individuals who carry the $9 \mathrm{R}$ allele, relative to $10 \mathrm{R}$ homozygotes, display greater VS activation during reward anticipation and receipt (Aarts et al, 2010; Dreher et al, 2009; Forbes et al, 2009). Further, nicotine-dependent 9R carriers display greater smoking cue-elicited VS activation (Franklin et al, 2009; 2011) and greater VS DA release after smoking (Brody et al, 2006).

Beyond the DAT1 VNTR, polymorphisms in other genes also affect DA tone and reward-related behavior, including AUD-specific phenotypes. Multilocus genetic composite scores comprised of genotypes at the DAT1 VNTR and polymorphisms in the genes encoding the DA-catabolizing enzyme catechol-O-methyltransferase (COMT) and the $\mathrm{D}_{2}$ and $\mathrm{D}_{4}$ receptors (DRD2 and DRD4) have been reported to predict striatal response to reward, such that individuals who carry a larger number of alleles putatively associated with high basal DA tone display greater reward-related VS activation (Nikolova et al, 2011; Stice et al, 2012). The polymorphisms aggregated in these composites include single-nucleotide polymorphisms (SNPs) in COMT (rs4680/val158met) and ANKK1 (rs1800497/Taq1A, originally believed to be in the adjacent $D R D 2$ promoter) and a $48 \mathrm{bp}$ VNTR in DRD4. The COMT met allele has been associated with reduced COMT efficiency (Chen et al, 2004), likely increasing extrasynaptic DA accumulation, and with heightened DA receptor sensitivity among AUD individuals (Schellekens et al, 2012). The ANKK1 Taq1A A1 allele has been associated with dysregulated DA response among AUD individuals (Schellekens et al, 2012) and is in high linkage disequilibrium with the DRD2 rs1076560 T allele, which has been associated with reduced striatal expression of the short (primarily presynaptic) $\mathrm{D}_{2}$ receptor isoform (Zhang et al, 2007), likely increasing extrasynaptic DA accumulation. Finally, the DRD4 VNTR long allele has been associated with reduced DRD4 mRNA expression (Simpson et al, 2010), altered intracellular signaling after $\mathrm{D}_{4}$ binding (Asghari et al, 1995), and, among heavy drinkers, greater alcohol craving after consumption of a priming drink (Hutchison et al, 2002), greater alcohol cue-elicited striatal activation (Filbey et al, 2008), and less cortical activation during response inhibition (Filbey et al, 2011).

Individually, each of these DA-related polymorphisms may also influence dopaminergic drug effects. Evidence is strongest for the DAT1 VNTR, which has been reported to moderate the effects of methylphenidate (Kasparbauer et al, 2015; Kooij et al, 2008), the $\mathrm{D}_{2}$ agonist bromocriptine (van Holstein et al, 2011), and the $\mathrm{D}_{2} / \mathrm{D}_{3}$ agonist ropinirole (MacDonald et al, 2016) on cognitive function. Among AUD individuals, we previously reported an interaction between DAT1 genotype and naltrexone, an opioid antagonist that decreases alcohol-induced striatal DA release, on alcohol cue-elicited medial prefrontal cortex activation (Schacht et al, 2013). Among individuals with schizophrenia, multiple studies suggest that DRD2 rs1076560 (Blasi et al, 2015) and COMT rs4680 (Schacht, 2016) moderate antipsychotic effects on schizophrenia symptoms. Finally, among heavy drinkers and AUD individuals, the DRD4 VNTR has been reported to moderate atypical antipsychotic (olanzapine) effects on drinking and cue-elicited alcohol craving (Hutchison et al, 2003, 2006), and opioid antagonist (LY2196044) effects on drinking (Wong et al, 2014). Taken together, these data strongly suggest the possibility of a pharmacogenetic interaction between DA-related genetic variation and dopaminergic drugs, particularly in AUD, in which DA function is dysregulated.

Given these findings, the current study investigated whether DAT1 VNTR genotype or a broader index of DArelated genetic variation comprised of genotypes at the DAT1 and DRD4 VNTRs and COMT rs4680 and DRD2 rs1076560 SNPs moderated APZ effects on reward-related phenotypes among non-treatment-seeking AUD individuals. Primary outcomes were alcohol cue-elicited VS activation and alcohol self-administration in a bar-lab setting. APZ has fewer adverse side effects than other atypical antipsychotics (Khanna et al, 2014), but its most common adverse effects are nausea, dizziness, and somnolence (Mallikaarjun et al, 2004); thus, adverse effect severity was a secondary outcome. $\mathrm{APZ}$, relative to placebo, was hypothesized to reduce all outcomes to a greater extent among DAT1 9R carriers, relative to $10 \mathrm{R}$ homozygotes, and among individuals with a larger number of alleles associated with relatively higher basal DA tone.

\section{MATERIALS AND METHODS}

\section{Overview}

The Medical University of South Carolina (MUSC) Institutional Review Board approved the study, which was an 8-day sub-acute dosing human laboratory investigation (ClinicalTrials.gov identifier: NCT01292057) conducted at MUSC between April 2011 and December 2015. All subjects provided informed consent before participation, for which they were compensated. After randomization to study medication, subjects underwent an fMRI alcohol cuereactivity task on day 7 of medication ingestion and a barlab paradigm on day 8 . To maximize cue reactivity and motivation to drink, they were instructed to abstain from alcohol on the evenings of days 6 and 7. These procedures were identical to those previously published (Anton et al, 2012; Schacht et al, 2013).

\section{Subjects}

Subjects were recruited via media advertisements and were required to be ages 21-40 and to meet DSM-IV (Diagnostic and Statistical Manual of Mental Disorders, revised 4th edition) diagnostic criteria for Alcohol Dependence, as assessed by the Structured Clinical Interview for DSM-IV (First et al, 2002). The upper age limit was chosen for two reasons. First, the study required alcohol administration; this can only be ethically achieved on an outpatient basis among non-treatment-seeking subjects, who tend to be younger than treatment seekers (Ray et al, 2017). Second, subjects were randomized to medication on the basis of a trait impulsivity measure (see below); as age and impulsivity are negatively correlated (Galvan et al, 2007), the upper limit was intended to ensure a sufficient number of individuals with 


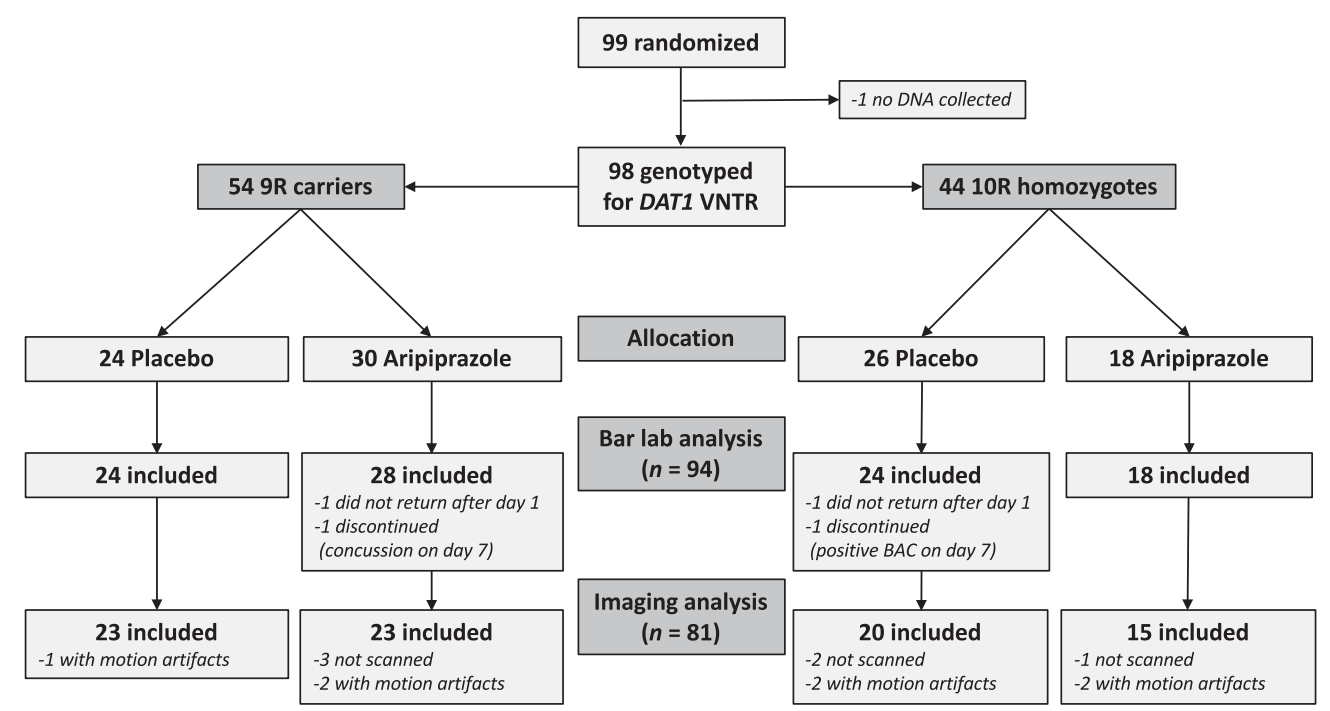

Figure I Subject flow through the study.

higher impulsivity. Exclusion criteria were as follows: current DSM-IV Substance Dependence for any other substance, except nicotine; current use of illicit substances or any prescription medication, as evidenced by urine drug screen and self-report; current DSM-IV Axis I diagnosis or suicidal/ homicidal ideation; history of significant medical illness; and liver enzyme (ALT or AST) levels greater than three times normal. Female subjects could not be pregnant or nursing. At baseline, the Alcohol Dependence Scale (Skinner and Allen, 1982), Obsessive Compulsive Drinking Scale (OCDS) (Anton et al, 1996), and Timeline Follow-back (Sobell and Sobell, 1992) were used to assess AUD severity, alcohol craving, and past-90-day drinking, respectively.

Figure 1 displays subject flow through the study. Ninetynine subjects were randomized to medication, but DNA was not collected from one subject. Of the remaining 98 subjects, 2 did not return after day 1 and 2 were discontinued from the study before completing the fMRI or bar-lab sessions, leaving 94 subjects for the bar-lab analysis (Table 1 lists demographic and severity data for these subjects). Of these individuals, 6 were not scanned, due to equipment issues $(n=2)$, claustrophobia $(n=2)$, and alcohol withdrawal symptoms on day $7(n=2)$, and 7 were excluded due to motion during the scan, leaving 81 subjects for the imaging analysis. Demographic and severity characteristics for this subset of subjects did not significantly differ from the larger sample.

\section{Genotyping}

Genomic DNA was extracted from peripheral blood mononuclear cells (Gentra Puragene Blood Kit; Qiagen, Valencia, CA) and PCR-amplified. VNTR genotypes were determined using custom primers 5'-TGTGGTGTAGGGAACGGCCTGAG-3' and 5'-CTTCCTGGAGGTCACGGCTCAAGG-3' (DAT1), and 5'-AGGACCCTCATGGCCTTG-3' and 5'GCGACTACGTGGTCTACTCG-3' (DRD4) (Thermo Fisher Scientific, Waltham, MA). Amplified samples were electrophoresed on $2.0 \%$ agarose gels and visualized with ethidium bromide under UV light, and genotypes were scored by two raters independently. For DAT1, two subjects carried alleles other than 9R or 10R: one had one 8R allele and one had two
$3 \mathrm{R}$ alleles. As alleles with $<9$ repeats have also been associated with reduced DAT expression relative to the $10 \mathrm{R}$ allele (Fuke et al, 2001), these alleles were categorized as 9R for analytic purposes. For DRD4, using the classification system most consistent in the addiction literature (McGeary, 2009), alleles were scored as "long" ( $\geqslant 7$ repeats) or "short" ( $<7$ repeats). DRD4 genotype could not be determined for one subject. SNP genotypes were determined with a StepOne Real-Time PCR System and Taqman 5'-nuclease assays (Thermo Fisher), using allele-specific probes (Catalog 4351376 and 4362691) and three known controls for each genotype. Genotypes for all polymorphisms (Table 2) were in Hardy-Weinberg equilibrium and consistent with expected population allele frequencies (Chang et al, 1996; Clarke et al, 2014; Kang et al, 1999; Palmatier et al, 1999).

\section{Randomization and Medication}

Subjects were urn randomized (Stout et al, 1994) to receive APZ (day 1: $5 \mathrm{mg}$; days 2-3: $10 \mathrm{mg}$; and days 4-8: $15 \mathrm{mg}$ ) or placebo for 8 days, instructed to take medication each morning, and were observed to ingest the first and last doses. APZ's elimination half-life is $\sim 60 \mathrm{~h}$ and peak concentration $\left(C_{\max }\right)$ for a $15 \mathrm{mg}$ dose occurs $\sim 3 \mathrm{~h}$ after ingestion (Mallikaarjun et al, 2004). Thus, medication was at $C_{\max }$ and $\sim 90 \%$ of steady state during the experimental procedures on days 7 and 8 . As subjects were non-treatment seekers who were not motivated to reduce their drinking, procedure timing was chosen to achieve near-maximal steady state while minimizing subject attrition that might have occurred by the time $100 \%$ steady state was achieved after 12-13 days of medication. Subjects and investigators were blind to both genotype and medication assignment. A parent study, described in a companion paper (Anton et al, 2017), hypothesized that APZ would reduce drinking more among subjects with greater trait impulsivity (briefly, data supported this hypothesis for bar-lab drinking). Thus, subjects were stratified by their baseline Barratt Impulsiveness Scale (BIS-11) (Patton et al, 1995) score into groups with BIS-11 scores greater vs. less than the median score of 68 observed in a previous pilot study (Voronin et al, 2008), 
Table I Demographic, Severity, and Drinking Data

\begin{tabular}{|c|c|c|c|c|c|c|}
\hline & \multicolumn{2}{|c|}{ DATI 9R carriers } & \multicolumn{2}{|c|}{ DATI IOR homozygotes } & \multirow[t]{2}{*}{$\boldsymbol{p}^{a}$} & \multirow[t]{2}{*}{$\boldsymbol{p}^{b}$} \\
\hline & Placebo & APZ & Placebo & APZ & & \\
\hline$N$ & 24 & 28 & 24 & 18 & - & - \\
\hline Age & $27.9(5.4)$ & $25.4(4.7)$ & $26.1(5.2)$ & $28.4(6.6)$ & 0.69 & 0.18 \\
\hline Sex (\% male) & 75.0 & 75.0 & 79.2 & 77.8 & 0.91 & 0.98 \\
\hline ADS score & I I.3 (6.8) & | $3.2(5.9)$ & $12.0(6.2)$ & $12.6(5.8)$ & 0.29 & 0.72 \\
\hline BIS-II score & $63.8(16.5)$ & $67.2(15.2)$ & $66.7(12.5)$ & $64.1(11.5)$ & 0.80 & 0.78 \\
\hline OCDS score & I $6.4(8.0)$ & | $8.0(8.6)$ & I6.3 (8.8) & $19.2(7.7)$ & 0.22 & 0.63 \\
\hline Drinks per day ${ }^{\mathrm{a}}$ & $6.2(2.8)$ & $8.6(3.9)$ & $7.2(2.8)$ & $7.8(2.9)$ & 0.02 & 0.05 \\
\hline Drinks per drinking day ${ }^{\mathrm{a}}$ & $7.9(3.1)$ & $10.7(3.4)$ & $9.5(3.6)$ & $9.3(3.4)$ & 0.04 & 0.04 \\
\hline
\end{tabular}

Abbreviations: ADS, Alcohol Dependence Scale; APZ, aripiprazole; BIS-I I, Barratt Impulsiveness Scale; OCDS, Obsessive Compulsive Drinking Scale.

Figures are means (SD) unless otherwise indicated. Current smoking was defined as smoking $\geqslant 10$ cigarettes per day. Statistics for differences between groups refer to the significance of the $\chi^{2}$-statistic for sex, race, and smoking, and the $t$ and $F$ statistics for other variables. $P$ values for significant differences $(p<0.05)$ are bolded.

$p^{a}=$ test for difference between medication groups.

$p^{b}=$ test for difference between all four groups.

aln the 90 days before medication randomization.

Table 2 Genotype Frequencies and Scoring for Each Polymorphism

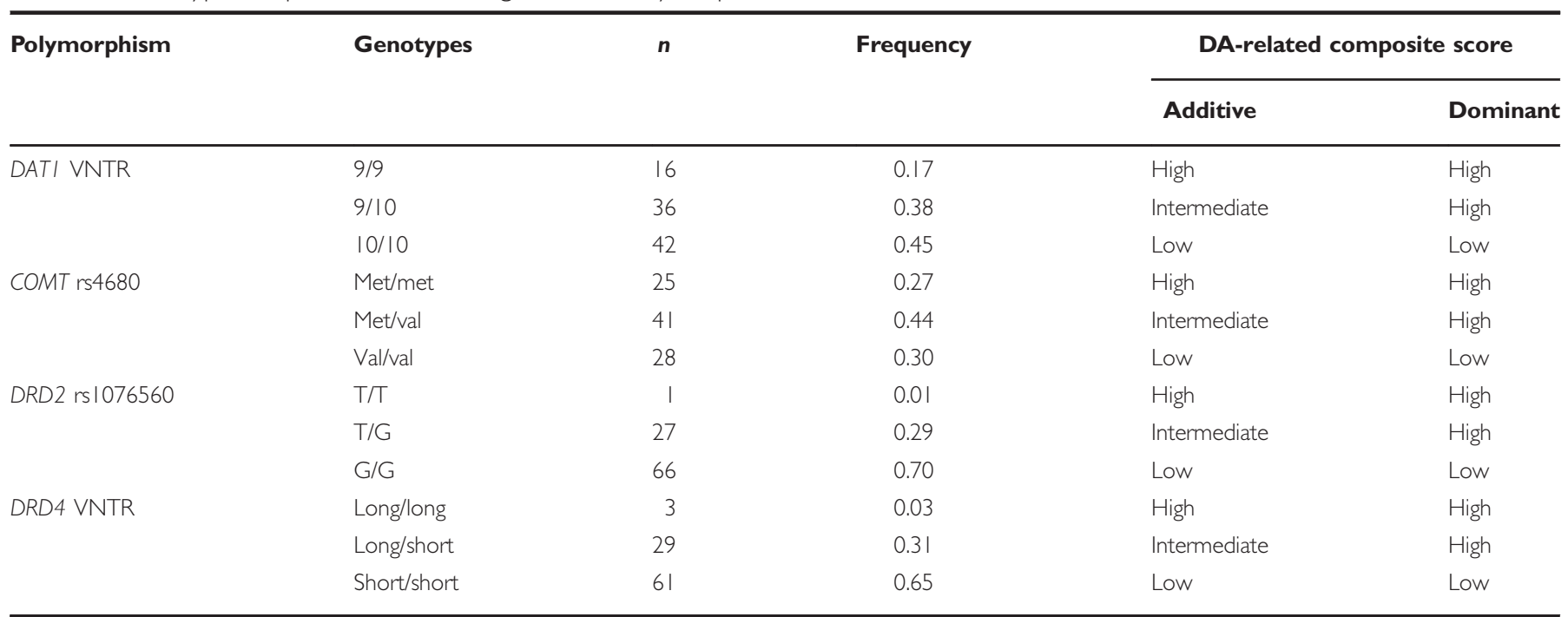

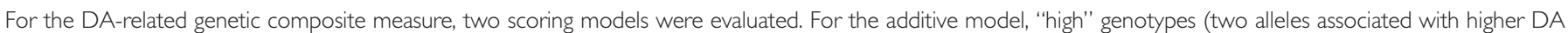

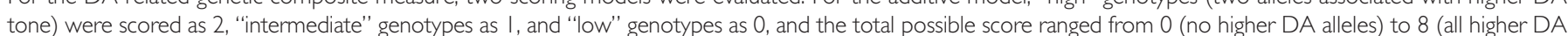

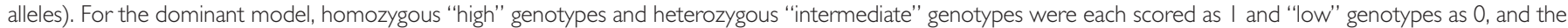
total possible score ranged from 0 (homozygous for all lower DA alleles) to 4 (homozygous or heterozygous for all higher DA alleles).

and randomization was conducted separately within each stratum. Urn variables balanced across medication groups within each stratum were sex and smoking status. Study medications were identically over-encapsulated with $100 \mathrm{mg}$ riboflavin and distributed in labeled blister packs. Urinary riboflavin was measured at baseline and on day 7 with a fluorometric assay based on standard curves of weighed-in riboflavin (Anton, 1996), and samples were considered adherent if the riboflavin value was $\geqslant 1000 \mathrm{ng} / \mathrm{ml}$ or had doubled since baseline.

\section{Neuroimaging}

On day 7, subjects were breathalyzed, assessed for alcohol withdrawal, and re-administered the OCDS. As noted above, one subject with a breath alcohol concentration $(\mathrm{BAC})>0$ and two subjects with Clinical Institute Withdrawal Assessment for Alcohol-Revised (Sullivan et al, 1989) scores $>4$ were excluded from scanning. Scans were scheduled in the afternoon or evening, to ensure medication was at $C_{\max }$. After acquisition of a high-resolution anatomical image, subjects were given a sip $(10 \mathrm{ml})$ of their preferred 80-proof liquor mixed with juice and administered a $12 \mathrm{~m}$-long task 
during which they passively viewed pseudorandomly interspersed blocks of alcoholic beverage images (ALC) (equally distributed between beer, wine, and liquor), non-alcoholic beverage images (BEV), blurred versions of these images that served as visual controls, and a fixation cross. Each 24 s-long block comprised only one image type and was followed by a $6 \mathrm{~s}$ period during which subjects were instructed to rate their urge for alcohol. Images were selected from a normative set (Stritzke et al, 2004), supplemented with images from advertisements, and matched for intensity, color, and complexity. This task consistently elicits robust cue-elicited VS activation among non-treatment-seeking AUD individuals (Schacht et al, 2011).

Functional images were acquired with a gradient echo, echo-planar imaging sequence implemented on a 3T TIM Trio scanner (Siemens, Erlangen, Germany). Acquisition parameters were as follows: repetition/echo times $=2200$ $/ 35 \mathrm{~ms} ; 328$ volumes; flip angle $=90^{\circ}$; field of view $=192 \mathrm{~mm}$; matrix $=64 \times 64$; voxel size $=3.0 \times 3.0 \mathrm{~mm} ; 36$ contiguous $3.0 \mathrm{~mm}$-thick transverse slices. Using FEAT (fMRI Expert Analysis Tool) v. 6.00, part of FSL (FMRIB's Software Library, Oxford) (Smith et al, 2004), functional images were realigned to the middle volume, spatially smoothed $(8 \mathrm{~mm}$ full width at half maximum kernel), resampled to $2 \mathrm{~mm}$ isotropic voxels, and registered, first to the subject's highresolution anatomical image and subsequently to the Montreal Neurological Institute (MNI) 152-subject-average template. Based on previous findings of greater reliability (Schacht et al, 2011) and better prediction of medication response (Schacht et al, 2017) for right, vs. left, cue-elicited VS activation, analyses focused on the right VS, defined $a$ priori as a $6 \mathrm{~mm}$-radius sphere centered at the point $(12$ $15-6)$ in MNI space. For each subject, this sphere was reverse registered from the MNI-152 image to the subject's anatomical image and the average percentage change of the blood oxygen level-dependent signal between ALC and BEV blocks (i.e., ALC vs. BEV percent signal change) was extracted using the FSL featquery tool.

\section{Bar-lab Paradigm}

On day 8, subjects were observed to ingest the last medication dose at $1130 \mathrm{~h}$. Thirty minutes later, they were provided a gender- and weight-adjusted standard caloric lunch. At $1400 \mathrm{~h}$, subjects were administered a priming drink (1:3 ratio of their preferred 80-proof liquor and juice), adjusted for gender, age, and weight to produce a targeted BAC of $30 \mathrm{mg} \%$ (Watson, 1989), and instructed to consume it within $5 \mathrm{~min}$. Forty minutes later, subjects were presented a tray of four drinks, each with a targeted BAC of $15 \mathrm{mg} \%$, and told they could consume as many as they desired over the next hour. After an hour, this tray was removed and another tray of four drinks was made available over a second hour. To create a decisional balance between drinking and abstaining, subjects were given a "bar credit" of $\$ 16$ with which to "purchase" drinks, at the cost of $\$ 2 /$ drink, and were told that any money they did not spend would be given to them the following day. After the procedure, subjects were given dinner and remained until $2200 \mathrm{~h}$. A BAC measurement below $20 \mathrm{mg} \%$ was required before departure and a friend or taxi drove subjects home.

\section{Adverse Effects}

A physical symptom checklist was used to assess the presence and severity (self-rated as none, mild, moderate, or severe) of 21 adverse effects at baseline and on day 8, immediately before the bar-lab paradigm.

\section{Statistical Analysis}

The general linear model (GLM) (SPSS 23, IBM, Armonk, NY) was used to test the interaction between medication and DAT1 genotype on the primary outcomes (VS ALC vs. BEV activation and the number of drinks consumed in the bar lab). A model that included between-subjects factors for medication, DAT1 genotype, and their interaction was tested for each outcome. Analyses first compared 9R carriers with 10R homozygotes and subsequently examined the additive effect of the $9 \mathrm{R}$ allele. By chance, baseline drinking significantly differed between groups, such that APZ-treated subjects, and specifically APZ-treated DAT1 9R carriers, drank more heavily (Table 1); accordingly, baseline drinks per day was covaried in all models. Significant interactions were followed up with simple effects contrasts. Effect sizes were estimated with the $\eta_{\mathrm{p}}^{2}$ statistic, which summarizes the amount of variance in the dependent variable attributable to the interaction term or simple effect, and for which effects can be categorized as "small" $\left(\eta_{\mathrm{p}}^{2} \geqslant 0.0099\right)$, "medium" $\left(\eta_{\mathrm{p}}^{2} \geqslant 0.0588\right)$, or "large" $\left(\eta_{\mathrm{p}}^{2} \geqslant 0.1379\right)$ (Cohen, 1969). VS activation was also analyzed for correlation (Pearson's $r$ ) with BIS scores, day 7 OCDS scores, and bar-lab drinking.

The GLM was also used to test the interaction between medication and a DA-related genetic composite on the primary outcomes. Based on previous data regarding the functional significance of each polymorphism and its consequences among AUD individuals, the DAT1 9R, COMT met, $D R D 2 \mathrm{~T}$, and $D R D 4$ long alleles were categorized as higher-DA alleles, and the total number of alleles each subject carried was calculated (Table 2). Few subjects carried more than four higher-DA alleles (four subjects had five alleles and one had seven), so these subjects were combined with those who carried four, yielding five groups of subjects with zero $(n=7)$, one $(n=15)$, two $(n=31)$, three $(n=22)$, or four or more $(n=18)$ alleles. These groups did not significantly differ on any characteristic indicated in Table 1 , except race (see below). A model that included betweensubjects factors for medication, the additive effect of the number of higher-DA alleles, and their interaction was tested for each outcome. To explore the contributions of each polymorphism relative to DAT1 genotype alone, every permutation of DAT1 genotype and the other polymorphisms (e.g., $D A T 1+C O M T, D A T 1+D R D 2, \quad D A T 1+C O M T$ $+D R D 2$, etc.) was calculated, and effect sizes for the interactions between these permutations and medication on the primary outcomes were estimated. A dominant model with two groups per polymorphism, in which higher DAallele homozygotes and heterozygotes were combined, was also tested; only three subjects had $\geqslant 1$ higher-DA allele for all four polymorphisms, so they were combined with those who carried $\geqslant 1$ allele for three polymorphisms.

For the secondary outcome (adverse effect severity), the main effect of medication on the presence/absence of each effect was first evaluated with the GLM. Insomnia, somnolence, irritability, trouble concentrating, nausea/vomiting, 
dizziness, fatigue, blurry vision, and difficulty reaching orgasm were significantly more frequent $(p<0.05)$ in the APZ group (see Anton et al, 2017 for detail). To account for the influence of these effects on the pharmacogenetic interactions evaluated above, the presence/absence of each was evaluated individually as a covariate in all models. To evaluate whether DAT1 genotype or DA composite score affected adverse effect severity, the generalized linear model was used to test interactions between medication and DAT1 genotype and between medication and the additive effect of the number of higher DA alleles on the severity of each effect that significantly differed between medication groups.

\section{RESULTS}

\section{Adherence}

Of the 94 subjects included in the bar-lab analysis, 36/47 (76.6\%) placebo-treated and 35/47 (74.4\%) APZ-treated subjects were deemed adherent by urinary riboflavin on day 7; these proportions were not significantly different $\left(\chi^{2}(1, N=94)=0.058, p=0.81\right)$.

\section{Cue-elicited VS Activation}

The interaction between medication and DAT1 genotype was significant $\left(\mathrm{F}(1,76)=4.98, p=0.029, \eta_{\mathrm{p}}^{2}=0.061\right)$. Relative to placebo, APZ reduced VS activation among 9R carriers, but increased it among 10R homozygotes (Figure 2a). The simple effect of medication approached significance among 10R homozygotes $(\mathrm{F}(1,76)=2.86, p=0.095)$. Baseline drinking did not significantly affect VS activation. In additive analyses, the interaction between medication and DAT1 genotype was also significant $(\mathrm{F}(1,76)=5.13, p=0.026$, $\left.\eta_{\mathrm{p}}^{2}=0.063\right)$, such that APZ, relative to placebo, reduced VS activation more among subjects with a greater number of $9 \mathrm{R}$ alleles. VS activation was significantly positively associated with day 7 OCDS score $(r(81)=0.26, p=0.018)$, but not BIS-11 score or bar-lab drinking.

\section{Bar-lab Drinking}

The main effect of medication $(\mathrm{F}(1,89)=4.63, p=0.034$, $\left.\eta_{\mathrm{p}}^{2}=0.049\right)$ and the interaction between medication and DAT1 genotype $\left(\mathrm{F}(1,89)=6.11, p=0.015, \eta_{\mathrm{p}}^{2}=0.064\right)$ were

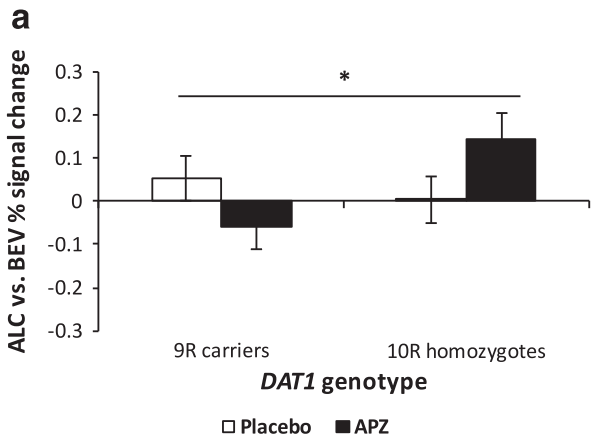

significant, such that APZ, relative to placebo, reduced drinking among 9R carriers, but not 10R homozygotes (Figure $2 \mathrm{~b}$ ). The simple effect of medication was significant only among $9 \mathrm{R}$ carriers $(\mathrm{F}(1,89)=11.54, p=0.001$, $\left.\eta_{\mathrm{p}}^{2}=0.115\right)$. Baseline drinking significantly affected bar-lab drinking $(\mathrm{F}(1,89)=4.80, p=0.031)$, such that subjects who drank more at baseline also drank more in the bar lab. In additive analyses, the interaction between medication and DAT1 genotype was also significant $(F(1,89)=4.73$, $\left.p=0.032, \eta_{\mathrm{p}}^{2}=0.050\right)$, such that $\mathrm{APZ}$, relative to placebo, reduced drinking more among subjects with a greater number of $9 \mathrm{R}$ alleles.

\section{DA-Related Genetic Composite Effects}

The interaction between medication and the additive effect of higher DA alleles across all four polymorphisms was significant for both VS activation $(\mathrm{F}(1,76)=4.12, p=0.046$, $\eta_{\mathrm{p}}^{2}=0.051$; Figure $\left.3 \mathrm{a}\right)$ and bar-lab drinking $(\mathrm{F}(1,88)=6.50$, $p=0.013, \eta_{\mathrm{p}}^{2}=0.069$; Figure $\left.3 \mathrm{~b}\right)$, such that APZ reduced these outcomes more among subjects with a greater number of higher DA alleles. The simple effect of medication on bar-lab drinking was significant only among subjects who carried four or more higher-DA alleles $(\mathrm{F}(1,82)=14.60, p=0.0005$, $\left.\eta_{\mathrm{p}}^{2}=0.151\right)$. Effect sizes for the pharmacogenetic interactions were larger for most genotype permutations than for DAT1 alone. For all interactions, APZ effects were better among individuals with a greater number of higher DA alleles. For VS activation, the largest effect was for the permutation of $D A T 1, D R D 2$, and DRD4 genotypes $\left(\eta_{\mathrm{p}}^{2}=0.084\right)$; for bar-lab drinking, for the permutation of DAT1, COMT, and DRD2 genotypes $\left(\eta_{\mathrm{p}}^{2}=0.10\right)$ (Table 3$)$. Interactions between the dominant model composite and medication were significant for both VS activation $\left(\mathrm{F}(1,76)=3.98, p=0.050, \eta_{\mathrm{p}}^{2}=0.050\right)$ and bar-lab drinking $\left(\mathrm{F}(1,88)=5.89, p=0.017, \eta_{\mathrm{p}}^{2}=0.063\right)$, such that APZ, relative to placebo, reduced these outcomes more among subjects with higher composite scores, with the largest effect in the ' $3+$ ' group.

\section{Impulsivity Effects}

As subjects were randomized to medication on the basis of their BIS-11 scores and we previously reported that BIS-11 self-control significantly moderated APZ effects on bar-lab drinking (Anton et al, 2017), we evaluated whether BIS-11

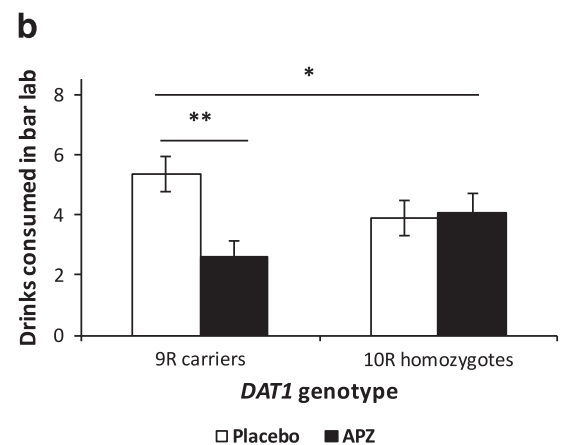

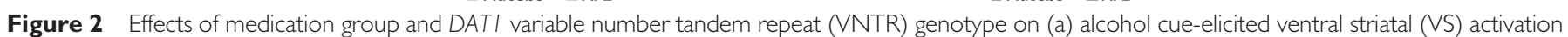

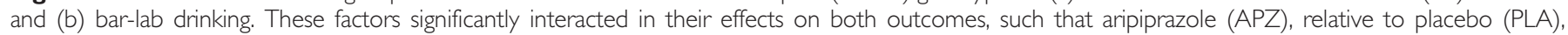

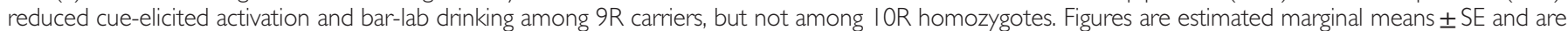

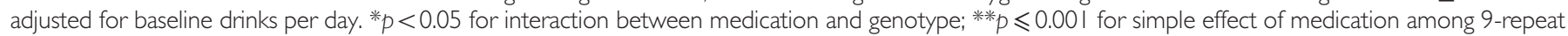
(9R) carriers. 
a

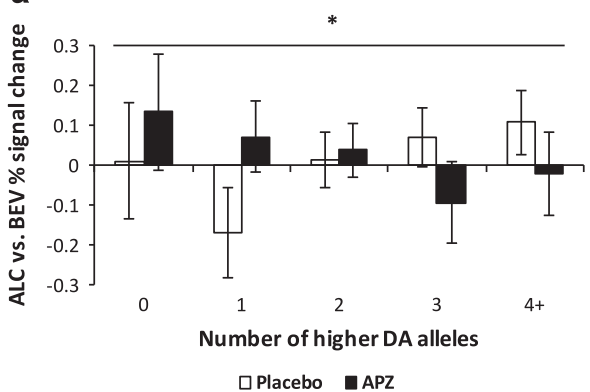

b

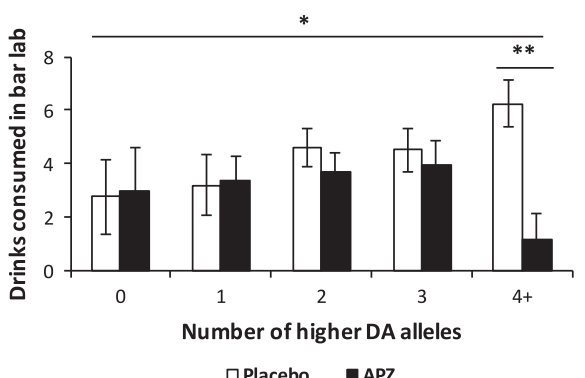

Figure 3 Effects of medication group and the additive dopamine (DA)-related genetic composite measure on (a) alcohol cue-elicited ventral striatal (VS) activation and (b) bar-lab drinking. These factors significantly interacted in their effects on both outcomes, such that aripiprazole (APZ), relative to placebo (PLA), reduced cue-elicited activation and bar-lab drinking more among subjects who carried a greater number of alleles associated with higher DA. Figures are estimated marginal means \pm SE and are adjusted for baseline drinks per day. $* p<0.05$ for interaction between medication and linear effect of number of higher DA alleles; ** $p \leqslant 0.00$ I for simple effect of medication among individuals with four or more higher DA alleles.

Table 3 P-values and Effect Sizes for Interactions Between Medication and Permutations of DATI VNTR and Other DARelated Polymorphisms

\begin{tabular}{|c|c|c|c|c|}
\hline \multirow[b]{2}{*}{ Genotype permutation } & \multicolumn{2}{|c|}{$\begin{array}{l}\text { VS activation } \\
\qquad(n=8 I)\end{array}$} & \multicolumn{2}{|c|}{$\begin{array}{l}\text { Bar lab drinks } \\
\quad(n=94)\end{array}$} \\
\hline & $p$ & $\eta_{p}^{2}$ & $p$ & $\eta_{\mathrm{p}}^{2}$ \\
\hline DATI & 0.026 & 0.063 & 0.032 & 0.050 \\
\hline DATI +COMT & 0.19 & 0.023 & 0.007 & 0.079 \\
\hline DATI+DRD2 & 0.021 & 0.068 & 0.006 & 0.081 \\
\hline$D A T I+D R D 4$ & 0.014 & 0.077 & 0.045 & 0.045 \\
\hline$D A T I+C O M T+D R D 2$ & 0.16 & 0.026 & 0.002 & 0.100 \\
\hline $\mathrm{DATI}+\mathrm{COMT+DRD} 4$ & 0.087 & 0.038 & 0.011 & $0.07 \mid$ \\
\hline$D A T I+D R D 2+D R D 4$ & 0.01 & 0.084 & 0.011 & 0.072 \\
\hline $\mathrm{DATI}+\mathrm{COMT+DRD2+DRD4}$ & 0.046 & 0.051 & 0.013 & 0.069 \\
\hline
\end{tabular}

Statistics are for the interaction between medication (aripiprazole vs. placebo) and each permutation of genotypes (additive effect of number of DATI 9R, COMT met, DRD2 T, and/or DRD 4 long alleles) in a general linear model that also included baseline drinks per day and the main effects of medication and the genotype permutation. $P$-values for significant interactions $(p<0.05)$ are bolded.

total or self-control subscale scores moderated the interactions between medication and either genetic factor (DAT1 genotype or the DA-related genetic composite) on VS activation or bar-lab drinking. In every model, the threeway interaction between the BIS-11 variable (total or selfcontrol score), the genetic factor, and medication was not significant, whereas the two-way interactions between each genetic factor and medication remained significant. Thus, pharmacogenetic effects did not vary as a function of impulsivity and these effects were independent from our previously reported interaction between self-control and APZ.

\section{Adverse Effects}

All interactions described above remained significant when any of the adverse effects that significantly differed between medication groups was covaried. The interaction between medication and DAT1 genotype on insomnia severity was significant (Wald $\left.\chi^{2}(1, N=94)=7.77, p=0.005\right)$, such that $10 \mathrm{R}$ homozygotes who received APZ, relative to placebo, had more severe insomnia, but $9 \mathrm{R}$ carriers did not. The interaction between medication and DAT1 genotype on irritability approached significance (Wald $\chi^{2}(1$, $N=94)=2.92, \quad p=0.088)$, in the same direction as the interaction for insomnia. DAT1 genotype did not significantly moderate the severity of any other effects that significantly differed between medication groups. There was also a significant interaction between medication and the additive effect of higher DA alleles on insomnia severity (Wald $\left.\chi^{2}(1, N=93)=5.01, p=0.025\right)$, but not other adverse effects, such that, among subjects who received APZ, insomnia was less severe among subjects with a greater number of higher DA alleles.

\section{Race Effects}

Consistent with expected allele frequencies, subjects with self-reported Caucasian ancestry ( $n=76$ for the total sample; $n=66$ for those with usable imaging data) were significantly more likely to have more higher DA alleles than nonCaucasian subjects $\left(\chi^{2}(1, N=93)=9.57, p=0.048\right)$, so all models were also evaluated among only Caucasian subjects. All effects that were statistically significant in the larger sample remained significant in this subsample, except for the interaction between medication and the genetic composite on VS activation, which was reduced to trend-level significance $(p=0.066)$.

\section{DISCUSSION}

Collectively, these data suggest a novel pharmacogenetic interaction between DA-related genetic variation and APZ response in AUD. This interaction was medium-sized, with large medication effects in the groups that responded better to APZ, and was present for both the DAT1 VNTR and a composite measure that aggregated genotypes at this VNTR and three other putatively functional DA-related polymorphisms. In each case, individuals with genotypes associated with higher basal DA tone displayed better APZ effects. Thus, APZ may be beneficial among individuals genetically predisposed to enhanced DA effects, but ineffective among others. This mixed profile could account for previous negative AUD clinical trial results.

As hypothesized, DAT1 9R carriers treated with APZ, compared with placebo, displayed less cue-elicited VS 
activation and alcohol self-administration, and reported less severe APZ-related insomnia, than 10R homozygotes. Given the 9R allele's association with lower DAT expression (Fuke et al, 2001) and enhanced reward-related VS activation (Aarts et al, 2010; Dreher et al, 2009; Forbes et al, 2009), these individuals might have been predisposed to greater striatal extrasynaptic DA accumulation or prolonged effects after alcohol cue exposure or the bar-lab priming drink. APZ's DA partial agonist effect might have displaced this elevated endogenous DA among these individuals, reducing DA-mediated cue reactivity or alcohol reward. As APZ, relative to placebo, also potentiated VS activation (which was associated with greater OCDS scores) among DAT1 10R homozygotes, its $\mathrm{D}_{2}$ partial agonist effect might have been detrimental among these individuals, who putatively have relatively lower DA tone. APZ's serotonergic effects might also have influenced this interaction; although beyond the scope of this work, evaluation of pharmacogenetic interactions between APZ and functional polymorphisms in serotonergic genes (e.g., SLC6A4 5-HTTLPR) might be fruitful. Although DAT1 genotype significantly moderated medication effects on both cue-elicited VS activation and bar-lab drinking, these outcomes were not significantly correlated, suggesting potentially dissociable effects. However, as bar-lab safety constraints limited the number of drinks available for self-administration, the upper range of this variable might have been artificially restricted, obviating correlation with (unrestricted) VS activation.

Beyond its interaction with DAT1 genotype, APZ, as hypothesized, also more effectively reduced VS activation and alcohol self-administration, and caused less severe insomnia, among individuals with a larger number of alleles associated with higher DA tone. Effect sizes for the pharmacogenetic interactions were larger for nearly all permutations of the genetic composite than for DAT1 alone, suggesting that the other DA-related polymorphisms accounted for additional variance in APZ effects. Individually, each of these polymorphisms has previously been reported to moderate DA partial agonist effects, with better effects noted among individuals carrying more higher-DA alleles (Blasi et al, 2015; Hutchison et al, 2003, 2006; Schacht, 2016). In contrast, an interaction in the opposite direction was reported between a similar DA-related genetic composite and the effects of the $\mathrm{D}_{2} / \mathrm{D}_{3}$ agonist ropinirole on impulsive decision-making, such that ropinirole, relative to placebo, increased impulsivity among healthy adults who carried more higher-DA alleles (MacDonald et al, 2016). Taken together with previous reports of greater rewardrelated VS activation among individuals who carried more higher DA alleles (Nikolova et al, 2011; Stice et al, 2012), these findings suggest that individuals genetically predisposed to high DA tone may be hypersensitive to reward, and that partial, but not full, DA agonists may reduce this hypersensitivity among these individuals, perhaps by normalizing DA tone.

Study strengths included low attrition and the use of wellvalidated alcohol cue reactivity and self-administration paradigms. However, several factors limit interpretation. First, subjects were younger, primarily male, non-treatmentseeking individuals who were compensated for participation. It is unclear whether these pharmacogenetic findings extend to individuals older than 40 years or to treatment seekers such as those examined in the multi-site APZ trial (Anton et al, 2008), and the number of females limited power to evaluate sex as a moderator of pharmacogenetic effects. Second, DA-related genetic variation was an exploratory aim and subjects were randomized to medication by their BIS-11 scores rather than DAT1 or other genotypes. However, impulsivity was well balanced between groups and did not significantly moderate pharmacogenetic effects. Finally, several caveats regarding the genetic composite should be noted. This measure assumed additive, rather than interactive, effects of its constituent polymorphisms, and combined polymorphisms associated with changes in both striatal and cortical DA. Low minor allele frequencies for the DRD2 and DRD4 polymorphisms precluded an interactive approach, which would have allowed evaluation of epistatic effects and of striatal vs. cortical DA effects on APZ efficacy. Previous work has suggested an interaction between COMT and DAT1 variation on reward-related VS activation (Dreher et al, 2009; Yacubian et al, 2007), but no two- or three-way interactions between $D A T 1, D R D 4$, and $D R D 2$ variation on this phenotype have been previously observed (Forbes et al, 2009). Previous studies of similar DA-related genetic composites have sometimes categorized "higher-DA" alleles differently for some polymorphisms. Notably, the DAT1 10R allele has been associated with lower DAT availability among controls and individuals with attention-deficit/hyperactivity disorder (Faraone et al, 2014). However, among AUD individuals, the 9R allele was associated with lower striatal DAT availability (Heinz et al, 2000). Nonetheless, further study of the functional consequences of these polymorphisms among AUD individuals would be valuable.

In conclusion, this study suggested that, among nontreatment-seeking young adults with AUD, polymorphisms in DAT1 and other DA-related genes moderated APZ effects on alcohol cue-elicited striatal activation and alcohol selfadministration. These findings require replication, but potentially support a precision medicine approach for APZ in AUD, in which DA-related genetic variation might be used to target this medication to individuals particularly likely to demonstrate better therapeutic effects and fewer adverse effects.

\section{FUNDING AND DISCLOSURE}

Dr. Schacht reports that, in the past 2 years, he has consulted for Laboratorio Farmaceutico CT. Dr. Anton reports that, in the past 2 years, he has consulted for Indivior, Xenoport, and Laboratorio Farmaceutico CT, and has received grant funding from Eli Lilly. $\mathrm{He}$ is the Chair of the Alcohol Clinical Trials Initiative (ACTIVE), which is conducted under the auspices of the American Society for Clinical Psychopharmacology and funded (currently or in the recent past) in part by Eli Lilly, Lundbeck, Pfizer, Ethypharm, Indivior, Xenoport/Arbor, Otsuka, and Amygdala. Drs. Randall and Voronin report no biomedical financial interests or potential conflicts of interest.

\section{ACKNOWLEDGMENTS}

We gratefully acknowledge Mark Ghent, B.A., for assistance with subject assessment and the bar-lab paradigm; Xingbao 
Li, M.D., and Scott Henderson, B.A., for assistance with fMRI data collection; and Yeong-bin Im, M.S., for performing the genotyping assays. This work was supported by grants from the National Institute on Alcohol Abuse and Alcoholism: K99/R00 AA021419 (JPS), K05 AA017435 (RFA), and P50 AA010761 (PI: Howard Becker). Portions of this work were presented at the 2016 American College of Neuropsychopharmacology meeting in Hollywood, FL, and the 2017 Research Society on Alcoholism meeting in Denver, CO. ClinicalTrials.gov identifier: NCT01292057 (https:// clinicaltrials.gov/ct2/show/NCT01292057).

\section{REFERENCES}

Aarts E, Roelofs A, Franke B, Rijpkema M, Fernandez G, Helmich $\mathrm{RC}$ et al (2010). Striatal dopamine mediates the interface between motivational and cognitive control in humans: evidence from genetic imaging. Neuropsychopharmacology 35: 1943-1951.

Anton RF (1996). New methodologies for pharmacological treatment trials for alcohol dependence. Alcohol Clin Exp Res 20(7 Suppl): 3A-9A.

Anton RF, Kranzler H, Breder C, Marcus RN, Carson WH, Han J (2008). A randomized, multicenter, double-blind, placebocontrolled study of the efficacy and safety of aripiprazole for the treatment of alcohol dependence. J Clin Psychopharmacol 28: 5-12.

Anton RF, Moak DH, Latham PK (1996). The obsessive compulsive drinking scale: A new method of assessing outcome in alcoholism treatment studies. Arch Gen Psychiatry 53: 225-231.

Anton RF, Schacht JP, Voronin KE, Randall PK (2017). Aripiprazole suppression of drinking in a clinical laboratory paradigm: influence of impulsivity and self-control. Alcohol Clin Exp Res 41: 1370-1380.

Anton RF, Voronin KK, Randall PK, Myrick H, Tiffany A (2012). Naltrexone modification of drinking effects in a subacute treatment and bar-lab paradigm: influence of OPRM1 and dopamine transporter (SLC6A3) genes. Alcohol Clin Exp Res 36: 2000-2007.

Asghari V, Sanyal S, Buchwaldt S, Paterson A, Jovanovic V, Van Tol $\mathrm{HH}$ (1995). Modulation of intracellular cyclic AMP levels by different human dopamine D4 receptor variants. J Neurochem 65: 1157-1165.

Blasi G, Selvaggi P, Fazio L, Antonucci LA, Taurisano P, Masellis R et al (2015). Variation in dopamine D2 and serotonin 5-HT2A receptor genes is associated with working memory processing and response to treatment with antipsychotics. Neuropsychopharmacology 40: 1600-1608.

Boileau I, Assaad J-M, Pihl RO, Benkelfat C, Leyton M, Diksic M et al (2003). Alcohol promotes dopamine release in the human nucleus accumbens. Synapse (New York, NY) 49: 226-231.

Brody AL, Mandelkern MA, Olmstead RE, Scheibal D, Hahn E, Shiraga $S$ et al (2006). Gene variants of brain dopamine pathways and smoking-induced dopamine release in the ventral caudate/ nucleus accumbens. Arch Gen Psychiatry 63: 808-816.

Chang FM, Kidd JR, Livak KJ, Pakstis AJ, Kidd KK (1996). The world-wide distribution of allele frequencies at the human dopamine D4 receptor locus. Hum Genet 98: 91-101.

Chen J, Lipska BK, Halim N, Ma QD, Matsumoto M, Melhem S et al (2004). Functional analysis of genetic variation in catecholO-methyltransferase (COMT): effects on mRNA, protein, and enzyme activity in postmortem human brain. Am J Hum Genet 75: 807-821.

Clarke TK, Weiss AR, Ferarro TN, Kampman KM, Dackis CA, Pettinati HM et al (2014). The dopamine receptor D2 (DRD2) SNP rs1076560 is associated with opioid addiction. Ann Hum Genet 78: 33-39.
Cohen J (1969). Statistical Power Analysis for the Behavioural Sciences. Academic Press: New York.

Dreher J-C, Kohn P, Kolachana B, Weinberger DR, Berman KF (2009). Variation in dopamine genes influences responsivity of the human reward system. Proc Natl Acad Sci USA 106: 617-622.

Faraone SV, Spencer TJ, Madras BK, Zhang-James Y, Biederman J (2014). Functional effects of dopamine transporter gene genotypes on in vivo dopamine transporter functioning: a metaanalysis. Mol Psychiatry 19: 880-889.

Filbey FM, Claus ED, Morgan M, Forester GR, Hutchison K (2011). Dopaminergic genes modulate response inhibition in alcohol abusing adults. Addict Biol 17: 1046-1056.

Filbey FM, Ray L, Smolen A, Claus ED, Audette A, Hutchison KE (2008). Differential neural response to alcohol priming and alcohol taste cues is associated with DRD4 VNTR and OPRM1 genotypes. Alcohol Clin Exp Res 32: 1113-1123.

First MB, Spitzer RL, Gibbon M, Williams JBW (2002). Structured Clinical Interview for DSM-IV-TR Axis I Disorders, Research Version, Non-patient Edition. Biometrics Research, New York State Psychiatric Institute: New York.

Forbes EE, Brown SM, Kimak M, Ferrell RE, Manuck SB, Hariri AR (2009). Genetic variation in components of dopamine neurotransmission impacts ventral striatal reactivity associated with impulsivity. Mol Psychiatry 14: 60-70.

Franklin TR, Lohoff FW, Wang Z, Sciortino N, Harper D, Li Y et al (2009). DAT genotype modulates brain and behavioral responses elicited by cigarette cues. Neuropsychopharmacology 34: 717-728.

Franklin TR, Wang Z, Li Y, Suh JJ, Goldman M, Lohoff FW et al (2011). Dopamine transporter genotype modulation of neural responses to smoking cues: confirmation in a new cohort. Addict Biol 16: 308-322.

Fuke S, Suo S, Takahashi N, Koike H, Sasagawa N, Ishiura S (2001). The VNTR polymorphism of the human dopamine transporter (DAT1) gene affects gene expression. Pharmacogenom $J \mathbf{1}$ : 152-156.

Galvan A, Hare T, Voss H, Glover G, Casey BJ (2007). Risk-taking and the adolescent brain: who is at risk? Dev Sci 10: F8-F14.

Heinz A, Goldman D, Jones DW, Palmour R, Hommer D, Gorey JG et al (2000). Genotype influences in vivo dopamine transporter availability in human striatum. Neuropsychopharmacology 22: 133-139.

Hutchison KE, McGeary J, Smolen A, Bryan A, Swift RM (2002). The DRD4 VNTR polymorphism moderates craving after alcohol consumption. Health Psychology: Official Journal of the Division of Health Psychology, American Psychological Association 21: 139-146.

Hutchison KE, Ray L, Sandman E, Rutter M-C, Peters A, Davidson $\mathrm{D}$ et al (2006). The effect of olanzapine on craving and alcohol consumption. Neuropsychopharmacology 31: 1310-1317.

Hutchison KE, Wooden A, Swift RM, Smolen A, McGeary J, Adler L et al (2003). Olanzapine reduces craving for alcohol: a DRD4 VNTR polymorphism by pharmacotherapy interaction. Neuropsychopharmacology 28: 1882-1888.

Ito H, Takano H, Arakawa R, Takahashi H, Kodaka F, Takahata K et al (2012). Effects of dopamine D2 receptor partial agonist antipsychotic aripiprazole on dopamine synthesis in human brain measured by PET with L-[beta-11C]DOPA. PLoS ONE 7: e46488.

Kang AM, Palmatier MA, Kidd KK (1999). Global variation of a 40bp VNTR in the 3'-untranslated region of the dopamine transporter gene (SLC6A3). Biol Psychiatry 46: 151-160.

Kasparbauer AM, Rujescu D, Riedel M, Pogarell O, Costa A, Meindl $\mathrm{T}$ et al (2015). Methylphenidate effects on brain activity as a function of SLC6A3 genotype and striatal dopamine transporter availability. Neuropsychopharmacology 40: 736-745.

Khanna P, Suo T, Komossa K, Ma H, Rummel-Kluge C, El-Sayeh $\mathrm{HG}$ et al (2014). Aripiprazole versus other atypical antipsychotics for schizophrenia. Cochrane Database Syst Rev CD006569. 
Kooij JS, Boonstra AM, Vermeulen SH, Heister AG, Burger $\mathrm{H}$, Buitelaar JK et al (2008). Response to methylphenidate in adults with $\mathrm{ADHD}$ is associated with a polymorphism in SLC6A3 (DAT1). Am J Med Genet B Neuropsychiatr Genet 147B: 201-208.

Kranzler HR, Covault J, Pierucci-Lagha A, Chan G, Douglas K, Arias AJ et al (2008). Effects of aripiprazole on subjective and physiological responses to alcohol. Alcohol Clin Exp Res 32: 573-579.

MacDonald HJ, Stinear CM, Ren A, Coxon JP, Kao J, Macdonald L et al (2016). Dopamine gene profiling to predict impulse control and effects of dopamine agonist ropinirole. J Cogn Neurosci 28: 909-919.

Mallikaarjun S, Salazar DE, Bramer SL (2004). Pharmacokinetics, tolerability, and safety of aripiprazole following multiple oral dosing in normal healthy volunteers. J Clin Pharmacol 44: 179-187.

Martinez D, Gil R, Slifstein M, Hwang DR, Huang Y, Perez A et al (2005). Alcohol dependence is associated with blunted dopamine transmission in the ventral striatum. Biol Psychiatry 58: 779-786.

McGeary J (2009). The DRD4 exon 3 VNTR polymorphism and addiction-related phenotypes: a review. Pharmacol Biochem Behav 93: 222-229.

Myrick H, Li X, Randall PK, Henderson S, Voronin K, Anton RF (2010). The effect of aripiprazole on cue-induced brain activation and drinking parameters in alcoholics. J Clin Psychopharmacol 30: 365-372.

Nikolova YS, Ferrell RE, Manuck SB, Hariri AR (2011). Multilocus genetic profile for dopamine signaling predicts ventral striatum reactivity. Neuropsychopharmacology 36: 1940-1947.

Oberlin BG, Dzemidzic M, Tran SM, Soeurt CM, Albrecht DS, Yoder KK et al (2013). Beer flavor provokes striatal dopamine release in male drinkers: mediation by family history of alcoholism. Neuropsychopharmacology 38: 1617-1624.

Palmatier MA, Kang AM, Kidd KK (1999). Global variation in the frequencies of functionally different catechol-O-methyltransferase alleles. Biol Psychiatry 46: 557-567.

Patton JH, Stanford MS, Barratt ES (1995). Factor structure of the Barratt impulsiveness scale. J Clin Psychol 51: 768-774.

Ray LA, Bujarski S, Yardley MM, Roche DJO, Hartwell EE (2017). Differences between treatment-seeking and non-treatmentseeking participants in medication studies for alcoholism: do they matter? Am J Drug Alcohol Abuse 43: 703-710.

Schacht JP (2016). COMT val158met moderation of dopaminergic drug effects on cognitive function: a critical review. Pharmacogenomics J 16: 430-438.

Schacht JP, Anton RF, Randall PK, Li X, Henderson S, Myrick H (2011). Stability of fMRI striatal response to alcohol cues: a hierarchical linear modeling approach. NeuroImage 56: 61-68.

Schacht JP, Anton RF, Voronin KE, Randall PK, Li X, Henderson S et al (2013). Interacting effects of naltrexone and OPRM1 and DAT1 variation on the neural response to alcohol cues. Neuropsychopharmacology 38: 414-422.

Schacht JP, Randall PK, Latham PK, Voronin KE, Book SW, Myrick $\mathrm{H}$ et al (2017). Predictors of naltrexone response in a randomized trial: reward-related brain activation, OPRM1 genotype, and smoking status. Neuropsychopharmacology 42: 2654.

Schellekens AF, Franke B, Ellenbroek B, Cools A, de Jong CA, Buitelaar JK et al (2012). Reduced dopamine receptor sensitivity as an intermediate phenotype in alcohol dependence and the role of the COMT Val158Met and DRD2 Taq1A genotypes. Arch Gen Psychiatry 69: 339-348.

Shapiro DA, Renock S, Arrington E, Chiodo LA, Liu LX, Sibley DR et al (2003). Aripiprazole, a novel atypical antipsychotic drug with a unique and robust pharmacology. Neuropsychopharmacology 28: $1400-1411$.

Simpson J, Vetuz G, Wilson M, Brookes KJ, Kent L (2010). The DRD4 receptor Exon 3 VNTR and 5' SNP variants and mRNA expression in human post-mortem brain tissue. Am J Med Genet B Neuropsychiatr Genet 153B: 1228-1233.

Skinner HA, Allen BA (1982). Alcohol dependence syndrome: measurement and validation. J Abnorm Psychol 91: 199-209.

Smith SM, Jenkinson M, Woolrich MW, Beckmann CF, Behrens $\mathrm{TE}$, Johansen-Berg $\mathrm{H}$ et al (2004). Advances in functional and structural MR image analysis and implementation as FSL. NeuroImage 23(Suppl 1): S208-S219.

Sobell LC, Sobell MB (1992)Timeline follow-back: a technique for assessing self-reported alcohol consumptionIn:Allen JP, Litten RZ (eds) Measuring Alcohol Consumption: Psychosocial and Biochemical Methods. Humana Press: Totowa, NJ. pp 41-72.

Stice E, Yokum S, Burger K, Epstein L, Smolen A (2012). Multilocus genetic composite reflecting dopamine signaling capacity predicts reward circuitry responsivity. J Neurosci 32: 10093-10100.

Stout RL, Wirtz PW, Carbonari JP, Del Boca FK (1994). Ensuring balanced distribution of prognostic factors in treatment outcome research. J Stud Alcohol Suppl 12: 70-75.

Stritzke WG, Breiner MJ, Curtin JJ, Lang AR (2004). Assessment of substance cue reactivity: advances in reliability, specificity, and validity. Psychol Addict Behav 18: 148-159.

Sullivan JT, Sykora K, Schneiderman J, Naranjo CA, Sellers EM (1989). Assessment of alcohol withdrawal: the revised clinical institute withdrawal assessment for alcohol scale (CIWA-Ar). $\mathrm{Br} J$ Addict 84: 1353-1357.

van Holstein M, Aarts E, van der Schaaf ME, Geurts DE, Verkes RJ, Franke B et al (2011). Human cognitive flexibility depends on dopamine D2 receptor signaling. Psychopharmacology (Berl) 218: 567-578.

Vergne DE, Anton RF (2010). Aripiprazole: a drug with a novel mechanism of action and possible efficacy for alcohol dependence. CNS Neurol Disord Drug Targets 9: 50-54.

Volkow ND, Wang G-J, Telang F, Fowler JS, Logan J, Jayne M et al (2007). Profound decreases in dopamine release in striatum in detoxified alcoholics: possible orbitofrontal involvement. $J$ Neurosci 27: 12700-12706.

Voronin K, Randall P, Myrick H, Anton R (2008). Aripiprazole effects on alcohol consumption and subjective reports in a clinical laboratory paradigm-possible influence of self-control. Alcohol Clin Exp Res 32: 1954-1961.

Watson PE (1989)Total body water and blood alcohol levels: Updating the fundamentalsIn:Crow KE, Batt RD(eds) Human Metabolism of Alcohol (Vol. 1): Pharmacokinetics, Medicolegal Aspects, and General Interest. CRC Press: Boca Raton.

Wong CJ, Witcher J, Mallinckrodt C, Dean RA, Anton RF, Chen Y et al (2014). A phase 2, placebo-controlled study of the opioid receptor antagonist LY2196044 for the treatment of alcohol dependence. Alcohol Clin Exp Res 38: 511-520.

Yacubian J, Sommer T, Schroeder K, Glascher J, Kalisch R, Leuenberger B et al (2007). Gene-gene interaction associated with neural reward sensitivity. Proc Natl Acad Sci USA 104: 8125-8130.

Yoder KK, Albrecht DS, Dzemidzic M, Normandin MD, Federici LM, Graves T et al (2016). Differences in IV alcohol-induced dopamine release in the ventral striatum of social drinkers and nontreatmentseeking alcoholics. Drug Alcohol Depend 160: 163-169.

Yokoi F, Grunder G, Biziere K, Stephane M, Dogan AS, Dannals RF et al (2002). Dopamine D2 and D3 receptor occupancy in normal humans treated with the antipsychotic drug aripiprazole (OPC 14597): a study using positron emission tomography and [11C] raclopride. Neuropsychopharmacology 27: 248-259.

Zhang Y, Bertolino A, Fazio L, Blasi G, Rampino A, Romano R et al (2007). Polymorphisms in human dopamine D2 receptor gene affect gene expression, splicing, and neuronal activity during working memory. Proc Natl Acad Sci U S A 104: 20552-20557. 